\title{
Quantitative analysis of movement of a cervical target during stereotactic radiosurgery using the Leksell Gamma Knife Perfexion
}

\author{
Laboratory investigation
}

\author{
Daniel Tonetti, M.S., ${ }^{1}$ Jagdish Bhatnagar, Sc.D., ${ }^{2}$ And L. Dade Lunsford, M.D. ${ }^{3,4}$ \\ Departments of ${ }^{2}$ Radiation Oncology and ${ }^{3}$ Neurological Surgery, and ${ }^{4}$ Center for Image-Guided \\ Neurosurgery, ${ }^{1}$ University of Pittsburgh School of Medicine, Pittsburgh, Pennsylvania
}

\begin{abstract}
Object. The design of the Leksell Gamma Knife Perfexion facilitates stereotactic radiosurgery (SRS) on cervical spine targets provided that the target itself is located superior to the standard $\mathrm{G}$ stereotactic head frame base ring and does not move. This study was designed to measure potential deviations of targets in the upper cervical spine while using the currently available Leksell Coordinate Frame G.

Methods. A commercially available skull-and-cervical spine model was adapted for SRS using the Leksell Gamma Knife Perfexion. The Leksell Coordinate Frame G was attached to the model, and both CT and fluoroscopic imaging were performed to determine the potential for target deviation at standard Gamma Knife treatment angles of $70^{\circ}, 90^{\circ}$, and $110^{\circ}$. In addition, target deviations observed at various heights of the patient positioning table were analyzed using a pair of orthogonal fluoroscopic images obtained at a standard $90^{\circ}$ gamma angle and compared with target position as it relates to a reference bed height of $4.5 \mathrm{~cm}$.

Results. An examination of multiple radiopaque targets embedded in or affixed to the model showed target deviations ranging from as low as $3.53 \mathrm{~mm}$ at the medial occiput-C1 junction to $15.56 \mathrm{~mm}$ at the $\mathrm{C} 3-4$ level during $70^{\circ}$ extension. Target deviations at $110^{\circ}$ flexion relative to targets on a $90^{\circ} \mathrm{CT}$ scan included deviations ranging from $0.58 \mathrm{~mm}$ at the medial occiput-C1 junction to $13.32 \mathrm{~mm}$ at the medial $\mathrm{C} 3-4$ level.

Relative to targets observed at the Perfexion table height of $4.5 \mathrm{~cm}$, target deviation at a table height of $3 \mathrm{~cm}$ varied from 0.44 to $5.26 \mathrm{~mm}$. At a table height of $5.5 \mathrm{~cm}$, target deviation varied from 0.44 to $3.60 \mathrm{~mm}$, and at a maximum height of $5.8 \mathrm{~cm}$, target deviation varied from 0.62 to $4.30 \mathrm{~mm}$.

Conclusions. Target deviation grossly exceeded clinical tolerance and was greater the farther the distance between the cranial base and the cervical spine target. Simple and reproducible methods that allow SRS centers to immobilize the patient's cervical spine using the currently available model $\mathrm{G}$ head frame are necessary to increase the range of targets that can be treated safely using the Leksell Gamma Knife Perfexion.

(http://thejns.org/doi/abs/10.3171/2012.3.GKS1266)
\end{abstract}

KeY Words • Gamma Knife surgery • stereotactic radiosurgery • cervical spine

I N 1992, Kondziolka and Lunsford ${ }^{2}$ described the lower limit of SRS using the Leksell Gamma Knife as the foramen magnum. Technological advances have now facilitated the use of the Gamma Knife to treat neoplastic disease as far inferior as C-3 when a Hamilton-Lulu frame is used. ${ }^{1}$ Using the Leksell Gamma Knife Perfexion and the standard stereotactic head frame, the Leksell Coordinate Frame G, placed as far inferiorly on the head as possible, we can reliably perform MR imaging and CT coregistration as far inferiorly as C-4 to treat extracranial targets.

Because the neck is able to flex and extend, intraspinal or neck targets may move relative to fixed anatomical structures and the stereotactic frame. In contrast, in-

\footnotetext{
Abbreviations used in this paper: Oc = occiput; PPS = patient positioning system; $\mathrm{SRS}=$ stereotactic radiosurgery.
}

tracranial contents remain immobile relative to the head frame. The anatomical position of extracranial targets, such as meningiomas in the foramen magnum or upper cervical spine, must remain consistent between target imaging and radiation dose delivery using the Gamma Knife.

If one observes target movement greater than 1.0 $\mathrm{mm}$, a cervical spine immobilization device may be applied to facilitate accurate and safe SRS of targets in the upper cervical spine. A target movement of $1.0 \mathrm{~mm}$ was selected as the cutoff point in this study because it is of similar magnitude to the precision reported for immobilization devices. ${ }^{3,4}$ Target movement will likely be greatest at locations farthest from the point of attachment of the stereotactic frame. Thus, we anticipated larger target movement at more inferior targets.

In addition to movement of the target between imag- 
D. Tonetti, J. Bhatnagar, and L. D. Lunsford

ing and treatment, we hypothesized that the position of the cervical target may vary with the height of the patient positioning table (PPS). At the time of treatment, adjustments in the Perfexion PPS are made to improve patient comfort. Potential target movement associated with the height of the PPS must also be analyzed to determine whether this factor must be taken into account to ensure target immobilization before sites in the upper cervical spine can be treated using the Gamma Knife.

In this study we used a modification of a commercially available head-and-neck model to assess target movement in the upper cervical spine and cervicomedullary junction so that we could quantify the accuracy of treatment of these targets. Demonstration of target movement in the upper cervical spine has clinical relevance for patients harboring intraspinal lesions between the foramen magnum and the $\mathrm{C} 3-4$ junction.

\section{Methods}

\section{The Cervical Spine Model}

A commercially available plastic skull-and-cervical spine model (American 3B Scientific) was adapted for SRS using the Leksell Gamma Knife Perfexion (Elekta AB). Multiple Beekley Y-SPOT radiopaque pellets (Beekley Corp.) were affixed to the model to simulate targets; 1 posteromedial and 2 posterolateral targets were affixed at each intervertebral level from the $\mathrm{Oc}-\mathrm{C} 1$ junction to the C3-4 level. The posteromedial pellet at each intervertebral level was affixed to the inferior surface of the superior vertebra or, in the case of the $\mathrm{Oc}-\mathrm{C} 1$ posteromedial pellet, the inferior surface of the occiput as shown in Fig. 1. The posterolateral pellets were fused with heat into the posterolateral spinal cord between the lamina of the corresponding superior and inferior vertebrae to approximate intraspinal targets. The posterolateral Oc-C1 targets were similarly fused into the spinal cord superior to the lamina of $\mathrm{C}-1$ and inferior to the occiput.

We constructed a locking mechanism that was attached to the $\mathrm{G}$ head frame and the base of the head-andspine model so that the spine portion of the model could be held at the clinically relevant Perfexion gamma angles of $110^{\circ}$ flexion, $70^{\circ}$ extension, and $90^{\circ}$ neutral for stability during imaging (Fig. 2).

The Leksell model G stereotactic head frame was attached to the head-and-neck model using ear bars that have an additional hole located superiorly to the first (Fig. 3 ), thereby facilitating lowering of the base ring to the region of C-4. Axial 1.25-mm-slice CT imaging of the model was performed on 3 separate occasions. The CT images were uploaded to the Leksell GammaPlan (Elekta AB) treatment planning system. Stereotactic coordinates $(\mathrm{x}, \mathrm{y}$, and $\mathrm{z})$ of each Y-SPOT radiopaque pellet were obtained from the GammaPlan (Fig. 4). Coordinates were measured at the center-most pixel of each pellet on axial, coronal, and sagittal scans. After we had obtained stereotactic coordinates from the GammaPlan for each target at each gamma angle, an overall radial deviation was calculated for each target. The coordinates of targets on the $110^{\circ}$ flexion and $70^{\circ}$ extension scans were compared with

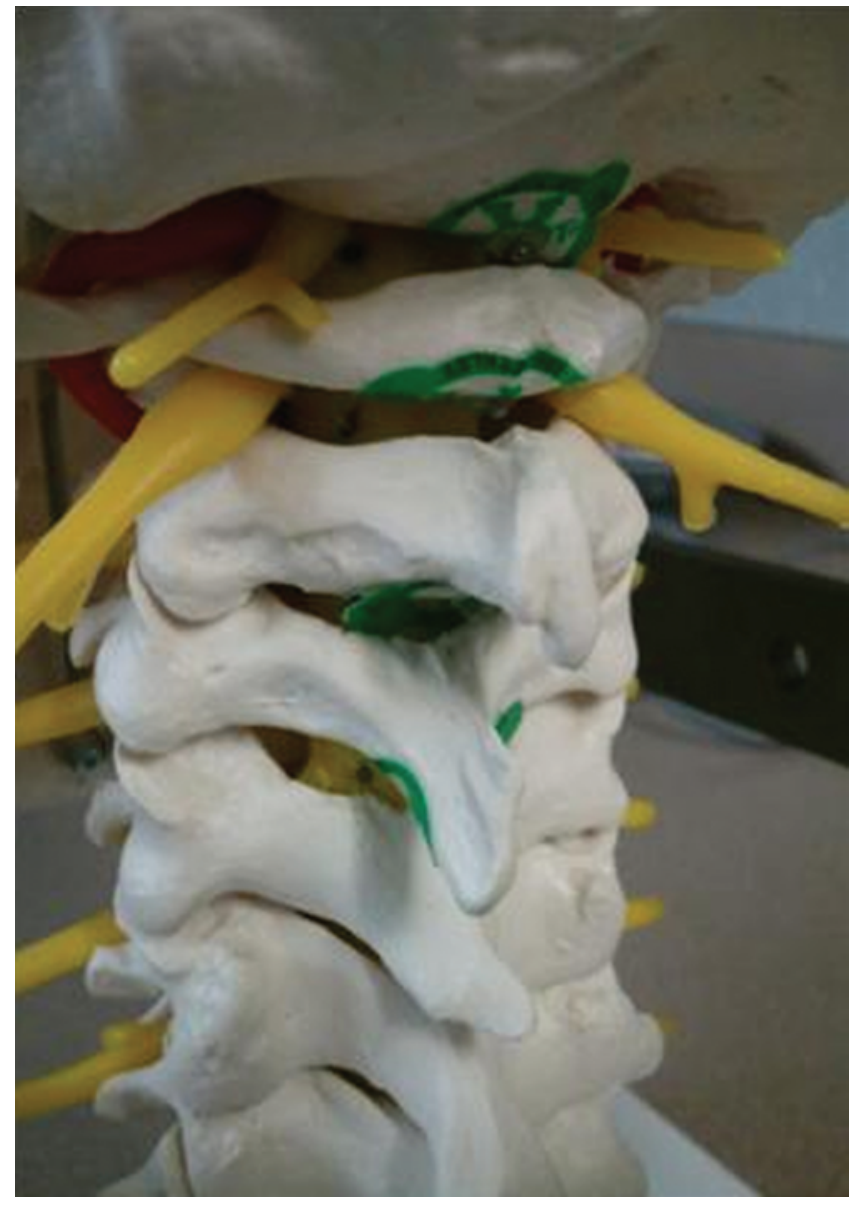

FıG. 1. Photograph depicting target placement on the head-andneck model. Multiple radiopaque targets were affixed to the model: 1 posteromedial and 2 posterolateral targets at each intervertebral level between $\mathrm{Oc}-\mathrm{C} 1$ and $\mathrm{C} 3-4$.

the same targets on the $90^{\circ}$ neutral scan. These calculations were repeated with imaging obtained on 3 separate days to obtain a quantitative measure of the precision of our measurements. The overall radial deviation of each target was calculated using the 3D analog of the Pythagorean theorem. In the equation below, values $\mathrm{x}_{1}, \mathrm{y}_{1}$, and $\mathrm{z}_{1}$ represent reference values obtained from the respective $90^{\circ}$ neutral scan.

$$
\text { deviation }=\sqrt{\left(x_{2}-x_{1}\right)^{2}+\left(y_{2}-y_{1}\right)^{2}+\left(z_{2}-z_{1}\right)^{2}}
$$

\section{Fluoroscopic Confirmation Related to the Height of the PPS}

In addition to target movement that happens between imaging and radiation delivery, we suspected that target movement could also occur when we adjusted the height of the PPS for the comfort of the patient. To measure the magnitude of target movement during this phase of the PPS adjustment, we employed a C-arm fluoroscopic $\mathrm{x}$ ray unit. Two orthogonal fluoroscopic views of the model were obtained for a variety of PPS heights after the model and $\mathrm{G}$ head frame were firmly docked to the PPS with 


\section{Quantitative analysis of cervical target movement during SRS}

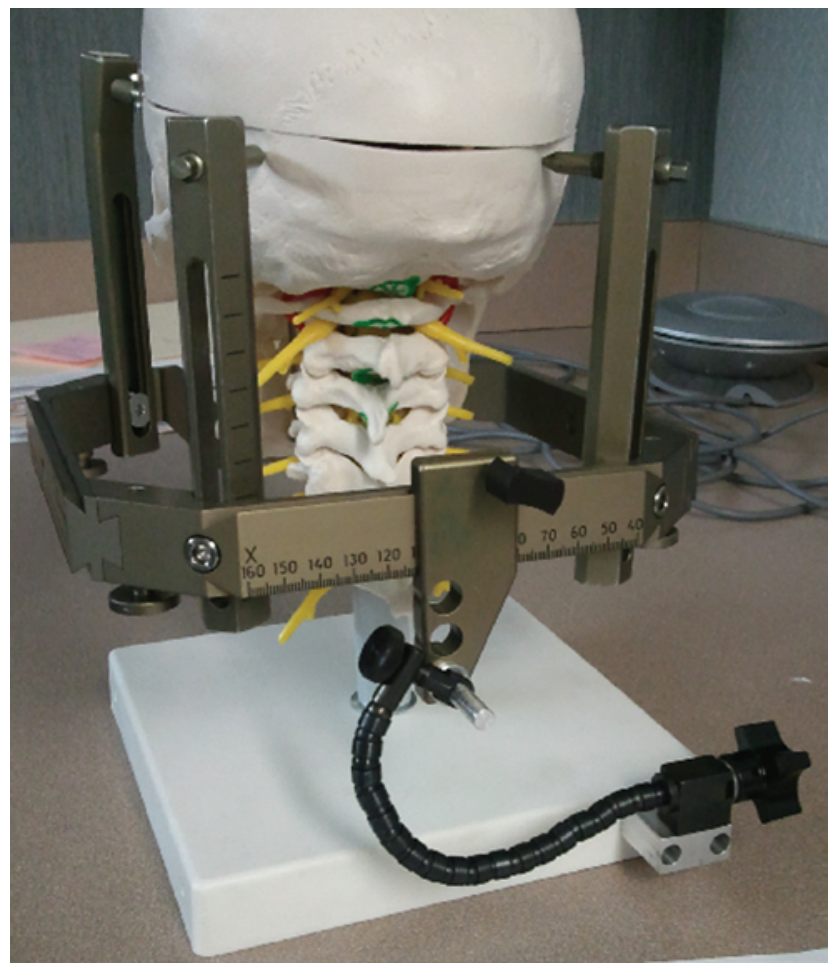

Fig. 2. Photograph showing a locking mechanism that was constructed between the base of the head-and-neck model and the $\mathrm{G}$ head frame to hold the model at $110^{\circ}$ flexion, $70^{\circ}$ extension, and $90^{\circ}$ neutral during imaging.

the frame adapter. The PPS height adjustment is fulcrum based, with the fulcrum near the middle of the PPS. Thus, when the PPS height is raised, the shoulder side of the PPS is raised, while the foot side of the PPS is lowered. We measured the height of the PPS at its head side, beginning with the highest possible setting and gradually decreasing it in $0.5-\mathrm{cm}$ steps until the model base could no longer be lowered. At each interval, fluoroscopic imaging was performed. The height of the PPS was measured using machinist-precision Vernier calipers. The magnification factor of the fluoroscopic images was determined at the target locations by knowing the actual diameter of the flexible metal gooseneck tubing used to represent the spinal cord in the model. Radial deviations associated with lowering and raising the PPS could be measured by referring to orthogonal fluoroscopic images, which were generated at each PPS height. A PPS height of $4.5 \mathrm{~cm}$ was used as the reference table height because it allows fluoroscopic visualization of targets at a neutral angle as the PPS is moved up and down. Only 4 targets were used for the calculation of radial target movement as a function of PPS height because the physical constraints of the Carm x-ray unit used for obtaining the 2 orthogonal views limited the visibility of other targets.

\section{Results}

Target Movement Related to Distance Below the Cranial Base

Radial target movement was assessed using CT im-

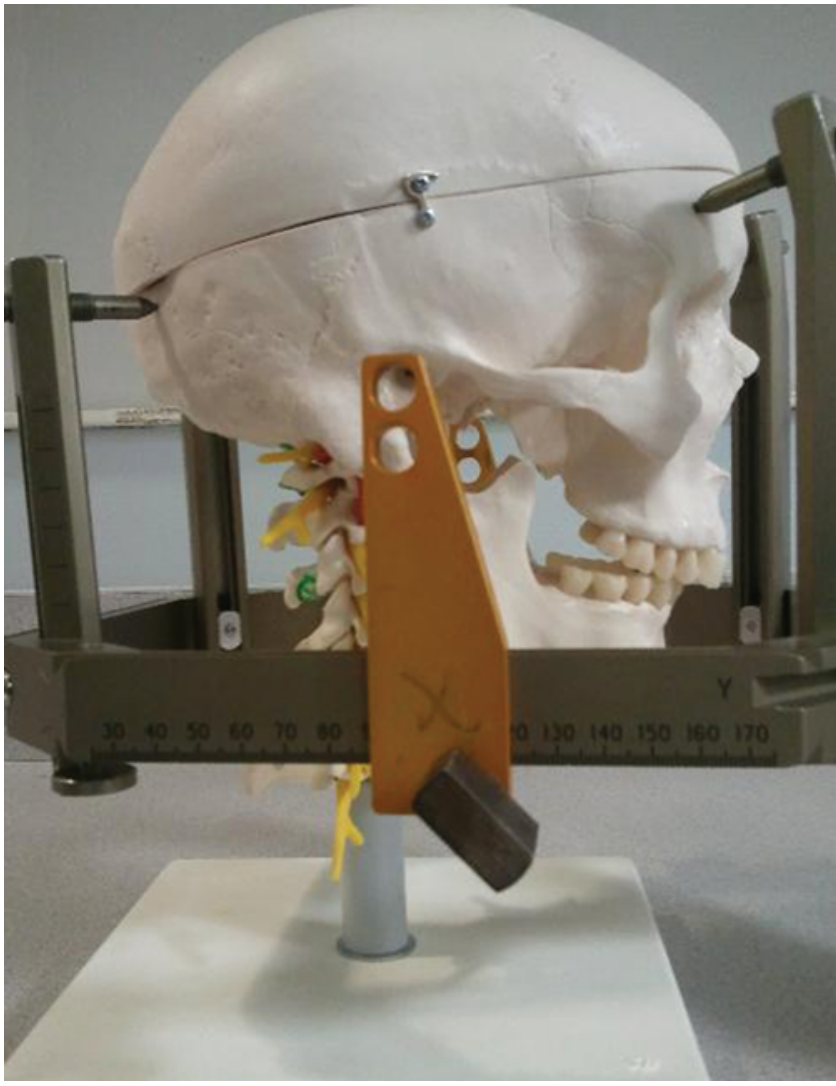

FIG. 3. Photograph demonstrating how the head frame was attached to the model using ear bars that have an additional hole located superiorly. The additional hole facilitates lowering of the base ring to the region of $\mathrm{C}-4$.

aging with respect to the neutral $90^{\circ}$ gamma angle. The results for the gamma angle of $70^{\circ}$ extension are shown in Table 1. We confirmed that average deviations increased as the distance between the cranial base and the target increased. At medial target positions the $\mathrm{Oc}-\mathrm{C} 1$ target moved $3.53 \pm 0.50 \mathrm{~mm}$, the $\mathrm{C} 1-2$ target moved $4.57 \pm$ $0.57 \mathrm{~mm}$, the $\mathrm{C} 2-3$ target moved $10.13 \pm 1.27 \mathrm{~mm}$, and the C3-4 target moved $15.56 \pm 1.98 \mathrm{~mm}$.

The CT images obtained at a $110^{\circ}$ flexion angle revealed a similar trend (Table 2), with increasing target deviations in inferiorly placed targets. At medial target positions the mean radial deviations were $0.58 \pm 0.15 \mathrm{~mm}$ at the $\mathrm{Oc}-\mathrm{C} 1$ target, $1.91 \pm 0.22 \mathrm{~mm}$ at the $\mathrm{C} 1-2$ target, $8.79 \pm 0.92 \mathrm{~mm}$ at the $\mathrm{C} 2-3$ target, and $13.32 \pm 1.44 \mathrm{~mm}$ at the C3-4 target.

\section{Target Movement Related to the Height of the PPS}

Radial target movement as a function of the PPS height was evaluated using a pair of orthogonal fluoroscopic images acquired by a C-arm fluoroscopic x-ray unit. A table height of $4.5 \mathrm{~cm}$ was used as the value to which target deviations at other table heights could be compared. The results are shown in Fig. 5. At the lowest height measured $-1.5 \mathrm{~cm}$ below the reference height of $4.5 \mathrm{~cm}$-the target deviation varied from $0.44 \mathrm{~mm}$ for the lateral $\mathrm{Oc}-\mathrm{C} 1$ target to $5.26 \mathrm{~mm}$ for the medial $\mathrm{C} 2-3$ target. At the maximum table height of $1.3 \mathrm{~cm}$ above the 


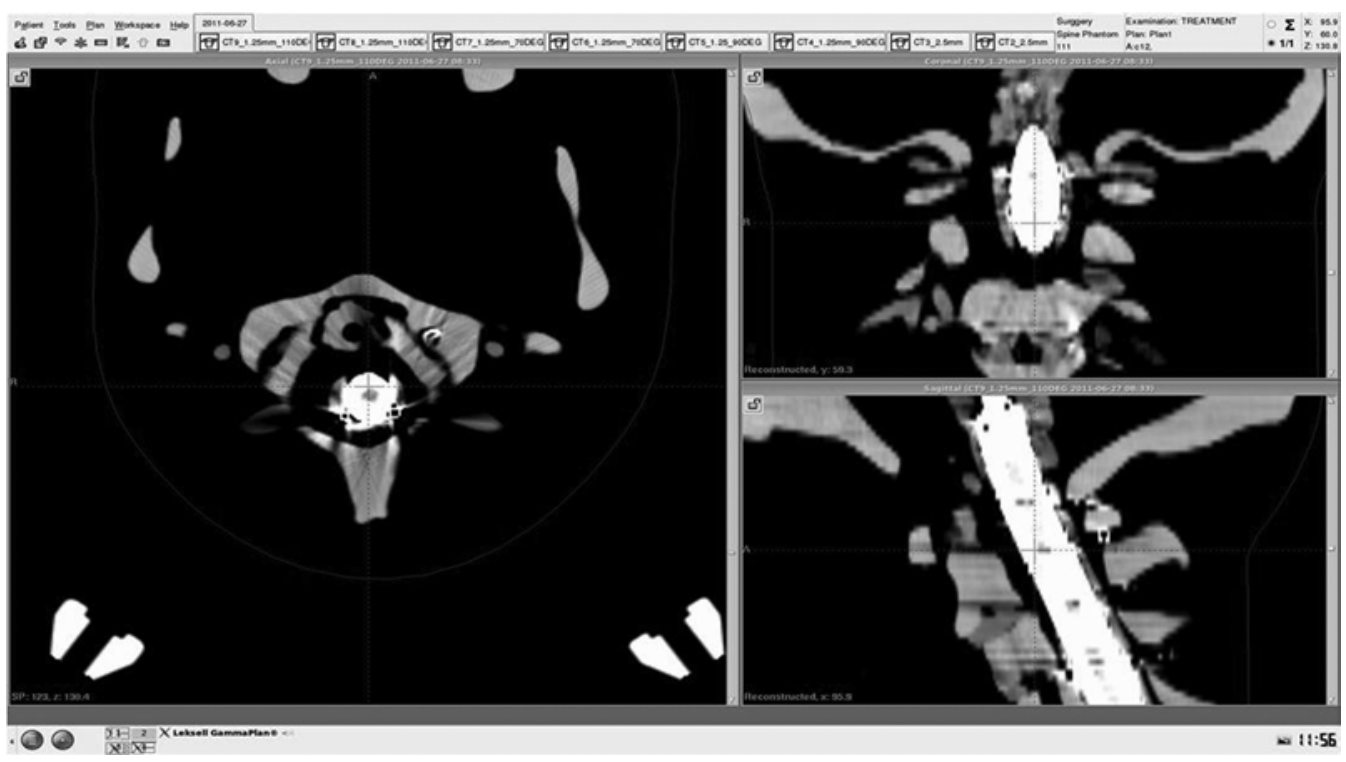

FIG. 4. Representative CT scans uploaded into Leksell GammaPlan software. Two posterobilateral (relative to the spinal cord) targets are visible on the axial scan (left) and one medial target is visible on the sagittal scan (lower right). Orthogonal coordinates were obtained using GammaPlan software, and flexion or extension angles were compared with a neutral $90^{\circ}$ angle to determine radial target movement.

reference height, the deviation ranged from $0.62 \mathrm{~mm}$ for the lateral Oc-C1 target to $4.30 \mathrm{~mm}$ for the medial $\mathrm{C} 2-3$ target. Lowering the PPS height by as little as $1.5 \mathrm{~cm}$ induced radial deviations of more than $5.2 \mathrm{~mm}$ at the $\mathrm{C} 2-3$ level.

\section{Discussion}

Accuracy is a major potential limiting factor for Gamma Knife surgery when the target is in the upper cervical spine ( $\mathrm{Cl}-4)$. Quantitative analysis of movement of such targets between the imaging and treatment stages of radiosurgery has not been previously reported. Here we show that there is the potential for target movement between the imaging and treatment stages of the SRS procedure. These deviations increase as the distance between

TABLE 1: Calculated target movement of the model at $70^{\circ}$ extension relative to $90^{\circ}$ neutral CT scans

\begin{tabular}{lcccr}
\hline & \multicolumn{4}{c}{ Target Movement (mm) } \\
\cline { 2 - 5 } Target Location & CT Scan 1 & CT Scan 2 & CT Scan 3 & Mean \pm SD \\
\hline rt lat Oc-C1 & 3.35 & 2.74 & 2.66 & $2.91 \pm 0.38$ \\
It lat Oc-C1 & 3.98 & 3.40 & 3.08 & $3.49 \pm 0.46$ \\
medial Oc-C1 & 4.05 & 3.49 & 3.06 & $3.53 \pm 0.50$ \\
rt lat C1-2 & 7.17 & 5.87 & 5.72 & $6.25 \pm 0.80$ \\
It lat C1-2 & 7.48 & 6.28 & 5.81 & $6.52 \pm 0.86$ \\
medial C1-2 & 5.23 & 4.18 & 4.31 & $4.57 \pm 0.57$ \\
rt lat C2-3 & 11.76 & 9.57 & 9.44 & $10.26 \pm 1.30$ \\
It lat C2-3 & 12.31 & 9.95 & 9.93 & $10.73 \pm 1.37$ \\
medial C2-3 & 11.59 & 9.29 & 9.49 & $10.13 \pm 1.27$ \\
rt lat C3-4 & 17.43 & 14.02 & 14.06 & $15.17 \pm 1.96$ \\
It lat C3-4 & 17.31 & 13.66 & 14.22 & $15.06 \pm 1.97$ \\
medial C3-4 & 17.85 & 14.31 & 14.53 & $15.56 \pm 1.98$ \\
\hline
\end{tabular}

the cranial base and the cervical spine target increases. For safe treatment of lesions in this area using SRS, patients must remain immobilized between the time of imaging and that of radiation delivery to ensure accuracy.

Data obtained from our comparisons of images showing $70^{\circ}$ extension and $110^{\circ}$ flexion to $90^{\circ}$ neutral images demonstrated that a change in neck flexion or extension, which occurs when the gamma angle is adjusted, results in target deviations relative to the stereotactic frame. Clinically, this suggests that the patient must undergo treatment at the same angle used during imaging to avoid target movement. As predicted, the data also confirmed that inferior targets are associated with much larger movements during both extension (Fig. 6) and flexion (Fig. 7).

TABLE 2: Calculated target movement of the model at $110^{\circ}$ flexion relative to $90^{\circ}$ neutral CT scans

\begin{tabular}{lcccc}
\hline & \multicolumn{4}{c}{ Target Movement (mm) } \\
\cline { 2 - 5 } Target Location & CT Scan 1 & CT Scan 2 & CT Scan 3 & Mean \pm SD \\
\hline rt lat Oc-C1 & 3.14 & 3.41 & 2.91 & $3.15 \pm 0.25$ \\
It lat Oc-C1 & 2.65 & 3.40 & 2.94 & $3.00 \pm 0.38$ \\
medial Oc-C1 & 0.44 & 0.58 & 0.73 & $0.58 \pm 0.15$ \\
rt lat C1-2 & 4.64 & 5.68 & 4.73 & $5.02 \pm 0.58$ \\
It lat C1-2 & 4.30 & 5.61 & 5.13 & $5.01 \pm 0.66$ \\
medial C1-2 & 1.73 & 2.16 & 1.85 & $1.91 \pm 0.22$ \\
rt lat C2-3 & 7.22 & 8.96 & 7.94 & $8.04 \pm 0.87$ \\
It lat C2-3 & 7.69 & 10.01 & 8.40 & $8.70 \pm 1.19$ \\
medial C2-3 & 8.38 & 9.84 & 8.15 & $8.79 \pm 0.92$ \\
rt lat C3-4 & 11.17 & 13.52 & 11.81 & $12.17 \pm 1.21$ \\
It lat C3-4 & 11.37 & 14.32 & 11.32 & $12.34 \pm 1.72$ \\
medial C3-4 & 12.45 & 14.99 & 12.53 & $13.32 \pm 1.44$ \\
\hline
\end{tabular}




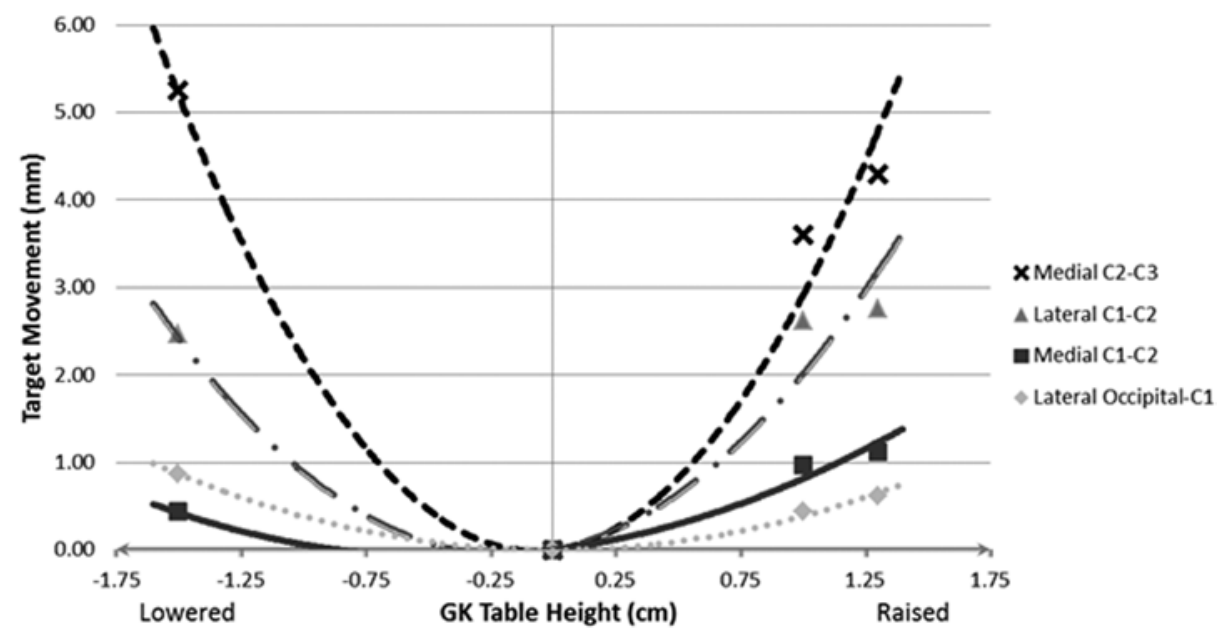

FIG. 5. Graph showing deviations of multiple targets as a function of Perfexion table height. A neutral table height of $4.5 \mathrm{~cm}$ (center square on $\mathrm{x}$ axis) was used as the reference height to which target movement at higher or lower table heights could be compared. Measurements on the $\mathrm{x}$ axis show variable table heights as they relate to the reference height. GK = Gamma Knife.

Our findings also confirmed that targets in the cervical spine move with respect to the stereotactic head frame as the Perfexion PPS is moved up or down. The numerical values for table height in this study are not important. The data show that regardless of the reference point, there is target movement as the PPS is altered. Therefore, all potential solutions to problems encountered with movement of the upper cervical spine during treatment with the Perfexion must keep the cervical spine immobilized as the PPS is adjusted.

Stereotactic radiosurgery using the Gamma Knife was initially developed to treat only intracranial lesions. With the redesign of the Gamma Knife and the creation of the current Perfexion model, the range of targets treatable by SRS has increased. Such targets include extracranial lesions of the head and neck region as well as cervical spine targets as far inferior as C-4. From a practical standpoint, the potential for the cranial vault to collide with the Perfexion collimator cap limits its use for targets lower than C-4. The current model G stereotactic frame is available throughout the world, and it represents the immobilization technique used for almost all SRS procedures involving the Gamma Knife. The Extend frameless stereotactic adaptation is marketed by the same manufacturer (Elekta AB) and is designed to facilitate fractionated radiotherapy of intracranial targets using the Perfexion. Other SRS manufacturers market different technologies that can be adapted to treat targets in the head and neck as well as the spine. Some of these products include the Accuray CyberKnife (Accuray Inc.), the Varian Trilogy (Varian Medical Systems), and the Elekta Synergy S or Axesse linear accelerator systems for frameless stereotactic body radiation. Depending on the system selected, innovative technologies that include robotics, immobilization body bags, and cone-beam CT imaging are available to evaluate and correct for potential target movement between the planning and treatment steps of SRS procedures.

\section{Future Solutions}

The presence of target movements relative to the stereotactic $\mathrm{G}$ head frame exceeds clinical tolerance and presents a barrier to safe and accurate treatment of targets in the upper cervical spine. One potential solution to this problem is intraoperative imaging-a solution that is likely to be effective yet expensive. An alternative simple solution involves using a commercially available orthotic brace, the Aspen CTO Cervical Collar (Aspen Medical Products), which immobilizes the cervical spine (Fig. 8). Use of this brace may allow centers to treat inferior targets reliably without any need for reengineering the currently widely available Leksell Coordinate Frame G. A simple cervical spine immobilization system may ultimately allow for accurate image acquisition, dose planning, and radiation delivery, thus expanding the range of targets suitable for Gamma Knife surgery.

\section{Weaknesses of the Current Study}

It is impossible for the phantom model used in this study to perfectly imitate a patient procedure. However, our data demonstrate that the potential for target move-

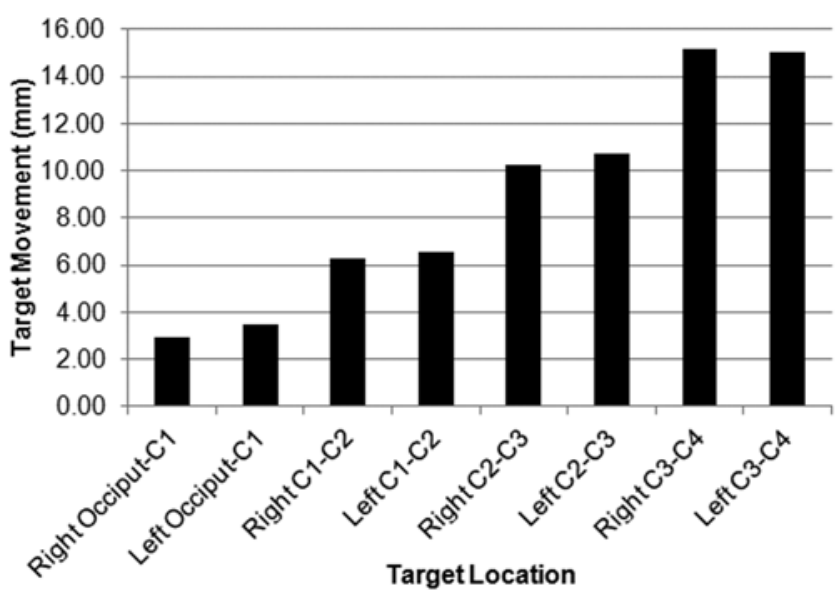

FIG. 6. Bar graph demonstrating mean posterolateral target movements during $70^{\circ}$ extension. Target deviation increases as targets are selected more inferiorly along the spine. 


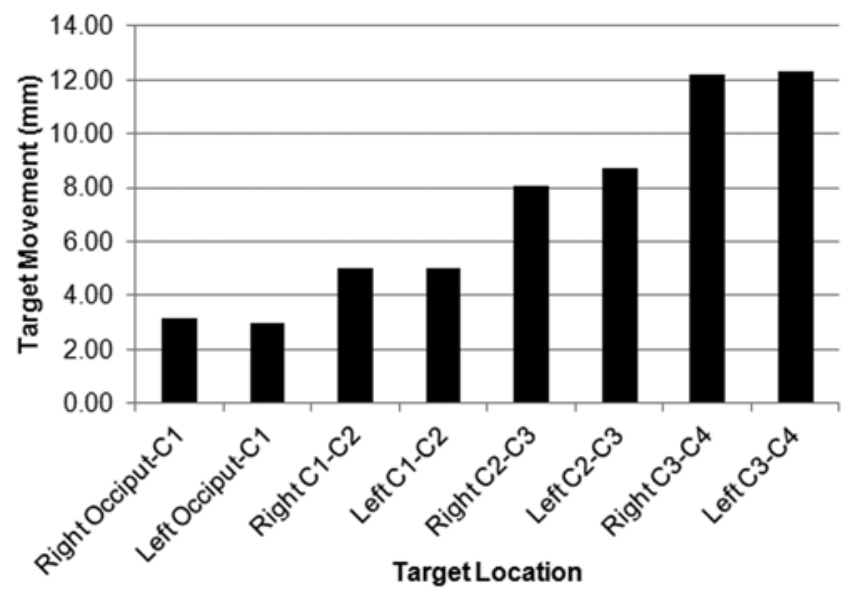

FIG. 7. Bar graph demonstrating mean posterolateral target movements during $110^{\circ}$ flexion. Target deviation increases as targets are selected more inferiorly along the spine.

ment between imaging and radiosurgery is real and therefore clinically significant. A limitation to this research is the fact that we did not quantify movement associated with rotation of the neck with respect to the stereotactic head frame. Further research is necessary to find a suitable solution that immobilizes the cervical spine and eliminates all movement-flexion, extension, and rotation-so that the patient can be treated accurately.

\section{Conclusions}

For lesions in the upper cervical spine, the stereotactic frame must be lowered as much as possible to allow the targeted lesion to approach the center of the collimator system. Using the standard $\mathrm{G}$ frame we can reliably obtain images as far inferiorly as C-4 using MR imaging

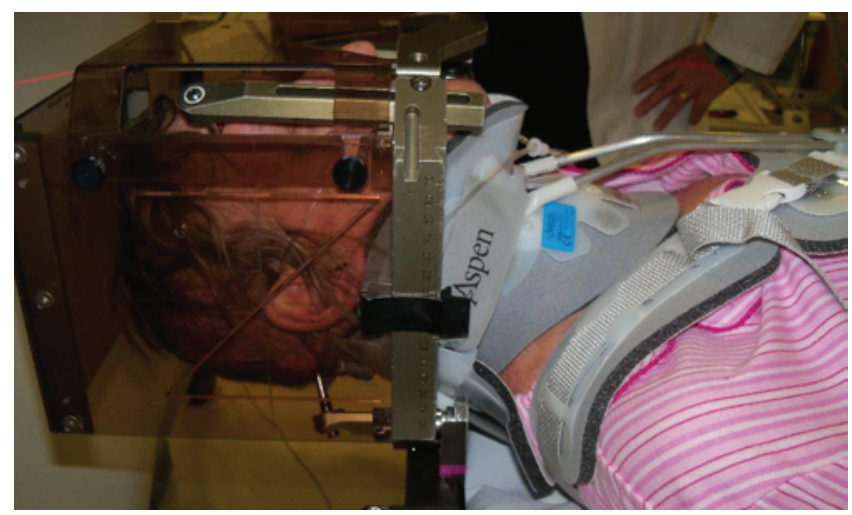

FIG. 8. Photograph showing a cervicothoracic orthotic brace that offers a potential simple solution to immobilizing the cervical spine during radiosurgery. Use of this brace may allow reliable treatment of inferior targets without the need for reengineering the Leksell stereotactic $G$ head frame. and CT coregistration. The target deviation for lesions between the occiput and C-4 grossly exceeds clinical tolerance and is greater at more inferior targets. Simple and reproducible methods that allow SRS centers to treat inferior targets reliably and safely without the need for reengineering the readily available $\mathrm{G}$ frame may increase the range of targets that can be treated effectively using the current model of the Leksell Gamma Knife Perfexion.

\section{Disclosure}

The work described in this report was funded by the Center for Image-Guided Neurosurgery at the University of Pittsburgh. Dr. Lunsford is a consultant for and stockholder in Elekta AB.

Author contributions to the study and manuscript preparation include the following. Conception and design: Lunsford, Tonetti. Acquisition of data: Tonetti, Bhatnagar. Analysis and interpretation of data: all authors. Drafting the article: Lunsford, Tonetti. Critically revising the article: all authors. Reviewed the submitted version of the manuscript: all authors. Approval of the final version of the manuscript on behalf of all authors: Lunsford. Statistical analysis: Lunsford, Tonetti. Study supervision: Bhatnagar.

\section{Acknowledgment}

The authors wish to express their gratitude to Mr. Al Ruckert of Hanger Prosthetics \& Orthotics (Pittsburgh, PA) for lending them the variously sized orthotic braces for trials involving healthy volunteers.

\section{References}

1. Hamilton AJ, Way D: Extracranial stereotactic radiosurgery, in Alexander E, Maciunas RJ (eds): Advanced Neurosurgical Navigation. New York: Thieme, 1999, pp 459-467

2. Kondziolka D, Lunsford LD: Radiosurgery of meningiomas. Neurosurg Clin N Am 3:219-230, 1992

3. Ruschin M, Nayebi N, Carlsson P, Brown K, Tamerou M, Li $\mathrm{W}$, et al: Performance of a novel repositioning head frame for gamma knife perfexion and image-guided linac-based intracranial stereotactic radiotherapy. Int J Radiat Oncol Biol Phys 78:306-313, 2010

4. Sweeney R, Bale R, Vogele M, Nevinny-Stickel M, Bluhm A, Auer T, et al: Repositioning accuracy: comparison of a noninvasive head holder with thermoplastic mask for fractionated radiotherapy and a case report. Int J Radiat Oncol Biol Phys 41:475-483, 1998

Manuscript submitted January 8, 2012.

Accepted March 22, 2012.

This report was presented at the 16th International Meeting of the Leksell Gamma Knife Society in Sydney, Australia, on March 27, 2012.

Please include this information when citing this paper: DOI: 10.3171/2012.3.GKS1266.

Address correspondence to: L. Dade Lunsford, M.D., University of Pittsburgh, Suite B-400, UPMC Presbyterian, 200 Lothrop Street, Pittsburgh, Pennsylvania 15213. email: lunsfordld@upmc.edu. 